\title{
Quantifying Fractal Dynamics of Human Respiration: Age and Gender Effects
}

\author{
C.-K. Peng, ${ }^{1}$ Joseph E. Mietus, ${ }^{1}$ Yanhui LiU ${ }^{2}$ Christine Lee, ${ }^{1}$ JefFrey M. HausdorfF, ${ }^{1,3}$ \\ H. EUGEne StAnley, ${ }^{2}$ ARY L. GOLDBERGER, ${ }^{1}$ and LEWIS A. LIPSITZ ${ }^{3,4}$ \\ ${ }^{1}$ Margret and H.A. Rey Institute for Nonlinear Dynamics in Medicine, Beth Israel Deaconess Medical Center, Harvard Medical \\ School, Boston, MA; ${ }^{2}$ Center for Polymer Studies and Department of Physics, Boston University, Boston, MA; \\ ${ }^{3}$ Gerontology Division of Beth Israel Deaconess Medical Center, Harvard Medical School, Boston, MA; and \\ ${ }^{4}$ Research and Training Institute of the Hebrew Rehabilitation Center for Aged, Boston, MA
}

(Received 28 December 2001; accepted 15 March 2002)

\begin{abstract}
We sought to quantify the fractal scaling properties of human respiratory dynamics and determine whether they are altered with healthy aging and gender. Continuous respiratory datasets (obtained by inductive plethysmography) were collected from 40 healthy adults (10 young men, 10 young women, 10 elderly men, and 10 elderly women) during 120 min of spontaneous breathing. The interbreath interval (IBI) time series were extracted by a new algorithm and fractal scaling exponents that quantify power-law correlations were computed using detrended fluctuation analysis. Under supine, resting, and spontaneous breathing conditions, both healthy young and elderly subjects had scaling exponents for the IBI time series that indicate long-range (fractal) correlations across multiple time scales. Furthermore, the scaling exponents (mean $\pm \mathrm{SD})$ for the IBI time series were significantly $(p<0.03)$ lower (indicating decreased correlations) in the healthy elderly male $(0.60 \pm 0.08)$ compared to the young male $(0.68 \pm 0.07)$, young female $(0.70 \pm 0.07)$, and elderly female $(0.67 \pm 0.06)$ subjects. These results provide evidence for fractal organization in physiologic human breathing cycle dynamics, and for their degradation in elderly men. These findings may have implications for modeling integrated respiratory control mechanisms, quantifying their changes in aging or disease, and assessing the outcome of interventions aimed toward restoring normal physiologic respiratory dynamics. (C) 2002 Biomedical Engineering Society. [DOI: 10.1114/1.1481053]
\end{abstract}

Keywords-Aging, Chaos theory, Long-range correlations, Respiration, Ventilation.

\section{INTRODUCTION}

Recent studies of cardiovascular dynamics have revealed fractal scaling in the organization of heart rate $^{16,17,29}$ and blood pressure ${ }^{18}$ fluctuations. The observed fractal scaling indicates the presence of longrange (power-law) correlations in the underlying dynamics. These long-range correlations seem to be a

Address correspondence to Ary L. Goldberger, MD, 330 Brookline Avenue, Boston, MA 02215. Electronic mail: agoldber@ caregroup.harvard.edu dynamical hallmark of integrative control systems. ${ }^{1}$ Moreover, in the case of heartbeat control, this organization degrades with aging and disease, ${ }^{16-18,29}$ suggesting a loss of coupling and functional integrity of the interacting physiologic control systems. In addition, a previous study ${ }^{32}$ provided evidence that heart rate dynamics may be less complex in men than women under certain conditions as assessed by an approximate entropy statistic.

Although neuroautonomic mechanisms that influence heart rate and blood pressure also affect, or are mediated by respiration, little is known about the possible longrange (fractal) organization of breathing patterns in adult humans, and the effects of aging and gender on respiratory variability. ${ }^{4,6,14,15,34}$ From a neurophysiologic viewpoint, such fractal behavior is of interest because it implies the presence of long-term dependence ("memory" effect). In the case of respiration, long-range correlations would mean that fluctuations in breathing rate would be correlated with respiratory variations hundreds of breaths earlier, and the correlation strength would decay in a scale-invariant (power-law) manner. ${ }^{2,10,29}$ Identification of such fractal correlation properties is important because it would guide ongoing efforts to develop realistic models of respiratory control. Furthermore, increased understanding of the organization of multiscale breathing patterns in health might be useful for the detection of subclinical disease, as well as the design of mechanical ventilators or other interventions to restore normal respiratory function.

In the present study, we sought to test the hypothesis that spontaneous breathing in humans, the output of a complex integrative control system, exhibits long-range (fractal) correlations. We also sought to assess quantitatively the possible effects of gender and physiological aging on these properties. A major methodologic difficulty impeding studies in this area is that the instantaneous lung volume signals recorded in the clinical labo- 
ratory are often very noisy and highly nonstationary. In order to study the long-range correlation properties of respiratory dynamics, we needed to (i) develop a reliable signal processing algorithm to accurately identify respiratory cycles in these noisy signals, and (ii) apply a scaling measurement technique that takes into account the intrinsic nonstationarities of the processed data.

In this study, we developed a robust algorithm for respiratory cycle detection and applied it to instantaneous lung volume signals recorded in a clinical laboratory. Then, we applied the detrended fluctuation analysis (DFA) technique ${ }^{28,29}$ to quantify the scaling behavior of these nonstationary respiratory time series. Based on the combination of these two algorithms, we present evidence indicating the presence of long-range correlations of breathing dynamics in healthy adults and a possible alteration of this behavior in elderly men.

\section{METHODS}

\section{Subjects}

Two groups of healthy adult subjects, 20 young (mean age 27, range 21-34 yr) and 20 elderly (mean age 74, range 68-81 yr), participated in this study. A study of heart rate dynamics for a subset of these subjects is reported elsewhere. ${ }^{17}$ Each group consisted of 10 women and 10 men. All subjects provided written informed consent and underwent a screening medical evaluation and electrocardiogram (ECG) to assure that they were in good health. The elderly subjects underwent a graded treadmill exercise tolerance test, to reduce the likelihood of undetected coronary artery disease.

\section{Respiratory Signal Acquisition}

Subjects lay supine for $120 \mathrm{~min}$ while continuous respiration signals were collected, using an inductive plethysmograph (Respitrace, Ambulatory Monitoring, Ardsley, NY). Signals were obtained from two elastic respiratory transducer bands, one placed around the midchest, and the other around the abdomen. All subjects remained in a resting, inactive state while watching the movie "Fantasia ${ }^{\Theta}$ " (Disney, Inc., 1940) in order to maintain wakefulness. The continuous respiration signals were digitized and sampled at $250 \mathrm{~Hz}$. The respiration signals were used as an indirect measurement of chest movement and lung volume. Since the physiologic focus of interest here is on fluctuations of the breath-to-breath cycle periods, it is necessary to map the respiration signal only to relative, not absolute, changes in lung volume. From each digitized respiration signal, the interbreath interval (IBI), i.e., the time period for one complete cycle of respiration (end-to-end inspiration) was determined via an automated computer program (see below) and visually verified. Each subject's IBI time

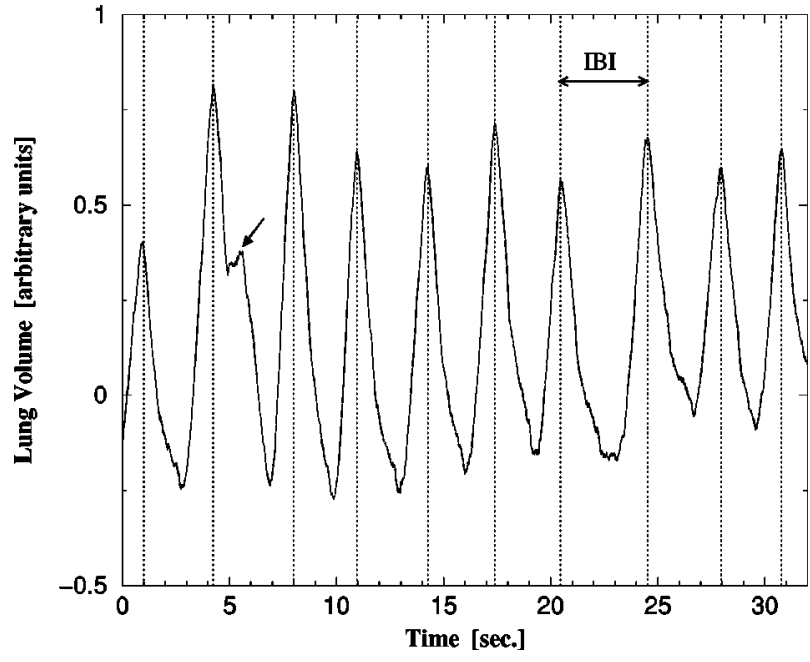

FIGURE 1. Representative segment of the digitized respiration signal illustrates the lung volume signal and the breathto-breath changes in the interbreath interval (IBI). The lung volumes are in arbitrary units. The dotted vertical lines denote the location of peaks detected by our algorithm. Note that the small bump (arrow) after the second peak is an example of a "type 2" local maximum discussed in the text. This local maximum is not likely a true respiration peak and was not identified as such by our algorithm.

series was then analyzed by detrended fluctuation analysis, ${ }^{28,29}$ a method designed to extract fractal scaling exponents from nonstationary time series, as well as by standard Fourier power spectrum analysis.

\section{Respiratory Signal Preprocessing}

There are two major problems related to the analysis of respiratory time series: (1) the signals are very complex, consisting of fluctuations related to intrinsic respiratory oscillations as well as external fluctuations due to environmental noise; and (2) the signals are highly nonstationary. These problems are illustrated in Fig. 1, which shows a representative digitized lung volume signal from a healthy elderly male subject. To study the respiratory dynamics from this noisy and nonstationary signal, robust preprocessing measures need to be developed to simplify the task of annotating the raw signals.

\section{Respiratory Signal Detection Algorithm}

Detecting the peak or trough of the respiration signal is the first step towards extracting the IBI time series. As shown in Fig. 1, since the signal near its trough becomes relatively flat, it is difficult to accurately identify the location of the minimum of each respiration signal. [These and all other original signals, as well as source codes of the analysis software from this study, will be made available via the Research Resource for Complex Physiologic Signals (http://www.physionet.org).] Therefore, we decided to use the peak-to-peak interval as the 
IBI. We tested available commercial software for peak detection and found that these software tools, which were developed for more general purposes, did not provide reliable detection for the types of noisy, nonstationary signals of interest here. Therefore, we developed a new algorithm to achieve this goal.

To accurately identify the peak of each respiration cycle, we need to find all the "local" maxima that correspond to the end points of inspiration. Two types of local maxima that are not true respiration peaks, however, are likely to be mistakenly identified by conventional peak-detection algorithms: (1) "type 1" false peaks are those local maxima caused by signal noise, and (2) "type 2" false peaks are caused by transient physiologic interruptions of the respiration signal. For example, in the expiration phase (with the respiration signal moving downward), there may be brief increases of lung volume creating local maxima in the signal (see Fig. 1). However, the overall movement of the signal is still dominated by a downward trend. If a transient upward bump is much smaller than the overall trend, then it is reasonable to assume that the expiration phase persists, and the local maxima should not be considered as a starting point of a new respiration cycle. Analogous pseudopeaks may also occur during the inspiration phase.

Although the mechanisms of the above two "false peaks" are different, their manifestations are similar. Therefore, we developed a unified approach to handle both cases. The basic concept of our algorithm is the following: We consider the respiration cycles to be alternating downward and upward trends in a noisy signal occasionally interrupted by relatively small reverse trends (introduced by brief pauses of breathing or other causes). Therefore, our task is to detect the basic downward and upward movements of the signal and the reversal of these trends.

To this end, we adapted the moving average analysis technique that has been widely used in economic time series analysis. ${ }^{5}$ The moving average over a window of width $W$ of a signal is defined as the average value of the signal at the preceding period of length $W$. To be more precise,

$$
X_{W}(t)=\frac{1}{W} \sum_{i=0}^{W-1} X(t-i),
$$

where $X$ is the signal, $t$ is the index of time, and $X_{W}$ is the moving average (of window $W$ ) of the signal.

Moving average processing reduces noise in the signal and facilitates detection of local trends. To discern local peaks, two moving averages are needed: one with a large window value $W_{1}$, and one with a small window value $W_{2}$. The longer-term moving average represents the slower trends and the shorter-term moving average re- flects fast-varying trends. We are interested in the crossover points of these two moving averages. When the short-term moving average crosses over to a value exceeding the long-term moving average, it indicates that the underlying signal is on an upward trend because the average of its recent history is greater than the average of its longer-term history. As the upward trend proceeds, the short-term moving average will diverge from the long-term moving average. The deviation of these two moving averages reaches a maximal value when the signal is around its peak. As the signal moves downward, the two moving averages approach each other, and eventually the short-term moving average crosses over to a value below the long-term moving average.

By carefully choosing the two window sizes $W_{1}$ and $W_{2}$, we effectively smooth out most of the local maxima generated by noise and pauses in breathing. In this study, we first examined a small subset of data to optimize the result of our algorithm. The optimized window sizes were set for $W_{1}=250$ (samples) and $W_{2}=25$ and applied to the complete database. Each true respiration peak is located between the crossover points where the shortterm moving average crosses over to values above and then below the long-term moving average curve. The "true" peak is identified simply as the largest local maximum between these two crossover points.

Our algorithm also takes into account the maximal deviation between the two moving averages. Because some signals might have a very large noise level, it is important to make sure that the crossover is not due to noise-related artifacts. To estimate the noise level locally, we calculate the standard deviation of the first difference of the signal in the observation window of width $W_{2}$. If the maximal deviation of the two moving averages is less than two times the standard deviation, then the second crossover point is very likely to be caused by noise and will be discarded by our algorithm.

After the algorithm automatically annotated the inspiratory peaks of the respiration cycles, we visually inspected the time series to correct misdetections (mostly overdetection of type 2 false peaks discussed above). Approximately $1 \%-2 \%$ of the peaks identified by the automated algorithm were corrected by this visual inspection. We also note that since it is difficult to unambiguously distinguish certain type 2 false peaks from true respiration peaks, we have systematically deleted all ambiguous peaks so that our approach is consistent. Our subsequent analysis is not very sensitive to a small amount of missing data.

\section{Time Series Analysis}

We investigated the difference in correlation properties of the respiratory rate time series between age and gender groups by applying a recently developed de- 

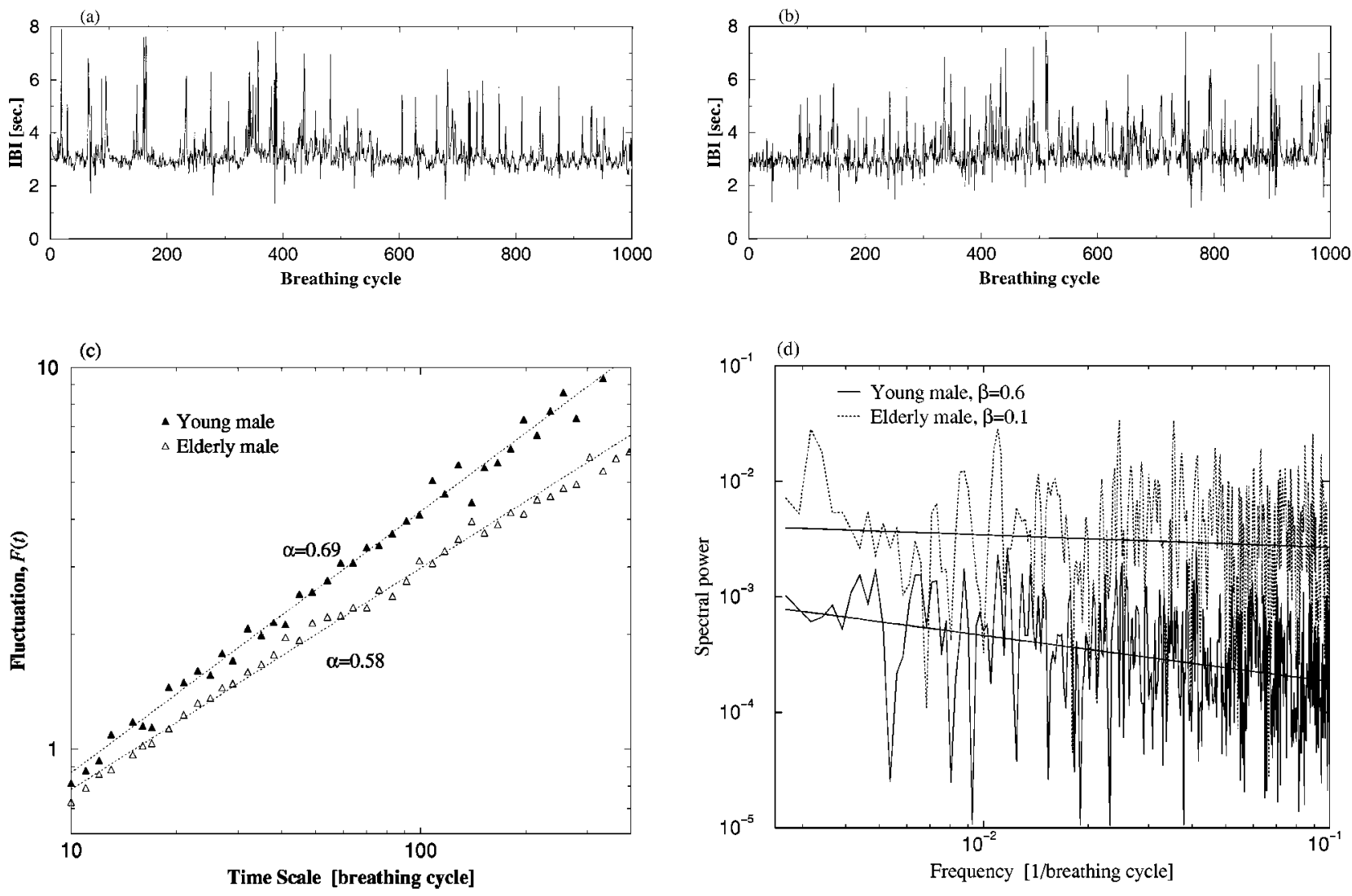

FIGURE 2. Illustrative respiratory time series for (a) young adult male and (b) elderly male. (c) Comparison of detrended fluctuation analysis (DFA) for subjects in (a) and (b) plotted on a double logarithmic scales. Dotted lines in panel (c) are the least-square fits for both subjects. The slopes of these fitted lines are the DFA exponents, $\alpha$. The slope for the elderly subject (closer to 0.5 ) is consistent with a degradation of long-range fractal scaling, indicating more random (uncorrelated) behavior. Fourier spectral densities are plotted in (d) for the same subjects in (a) and (b). The $\beta$ exponents are given by linear regression fits [solid lines in (d)]. Note that the noisy spectra lead to less reliable estimations of fractal scaling exponents compared with the DFA plots in (c). To facilitate comparison of scaling behavior in (d), we have offset the two sets of data points.

trended fluctuation analysis technique and traditional Fourier analysis. Figure 2 shows two typical IBI time series: one from an elderly subject [Fig. 2(a)], and the other from a young adult [Fig. 2(b)]. The respiratory rate (cycle per minute), $\varphi$, can be calculated from the IBI via the relation $\varphi=60 / I B I$. Our studies show that both the respiratory rate and IBI time series give very similar results. We present here our analysis of the IBI time series.

\section{Detrended Fluctuation Analysis}

One useful approach to study the scaling properties of a time series is to apply methods derived from the concept of self-similar (fractal) processes. The concept of self-similar processes was developed by Hausdorff, Besicovich, Kolmogorov, and Mandelbrot. ${ }^{19,25}$ An object is self-similar if its subsets can be rescaled to resemble (statistically) the original object itself. A scaling exponent (also called the self-similarity parameter) can be defined by this rescaling process. Long-range (power- law) correlations and self-similar processes are related in that a stationary time series with long-range correlations can be integrated, i.e., by forming an accumulated sum, to generate a self-similar process. Therefore, measurement of the self-similarity scaling exponent of the integrated time series can reveal long-range correlation properties of the original time series. Hurst analysis ${ }^{2}$ and root-mean-square analysis of random walks ${ }^{26}$ are both based on this concept.

To minimize the effect of nonstationary trends present in "real-world" physiologic data, we applied a previously validated method-termed detrended fluctuation analysis $^{28,29}$ - to the analysis of the respiratory rate. The advantages of DFA over conventional methods (e.g., spectral analysis and Hurst analysis) are that it permits the detection of long-range correlations embedded in nonstationary time series, and also avoids the spurious detection of apparent long-range correlations that are artifacts of nonstationarities. This method has been validated on control time series that consist of long-range 
correlated data superimposed on a nonstationary external trend. ${ }^{28}$ This method has been successfully applied to detect long-range correlations in highly complex heart beat time series, ${ }^{12,17,24,29}$ and other physiological signals. ${ }^{8-11}$

A detailed description of the DFA algorithm appears elsewhere. ${ }^{28,29}$ Briefly, the IBI time series (with $N$ data points) is first integrated, $y(k)=\sum_{i=1}^{k}\left[I(i)-I_{\text {ave }}\right]$, where $I(i)$ is the IBI at time $i$ and $I_{\text {ave }}$ is the average IBI. Next, the integrated time series is divided into boxes of equal length, $n$. In each box of length $n$, a least-squares line segment is fit to the data (representing the trend in that box). The $y$ coordinate of the straight line segments is denoted by $y_{n}(k)$. Next, we detrend the integrated time series, $y(k)$, by subtracting the local trend, $y_{n}(k)$, in each box. The root-mean-square fluctuation of this integrated and detrended time series is

$$
F(n) \equiv \sqrt{\frac{1}{N} \sum_{k=1}^{N}\left[y(k)-y_{n}(k)\right]^{2}} .
$$

One calculates $F(n)$ for a range of time scales (box sizes) to provide a relationship between $F(n)$, the average fluctuation as a function of box size. In our case, the box size $n$ ranged from 10 respiration cycles (about $40 \mathrm{~s}$ ) to 400 cycles (about $26 \mathrm{~min}$ ). The lower cutoff scale of $n$ is selected such that the artifact of the DFA, which appears on a small scale, will be minimized. ${ }^{3}$ While the upper bound of $n$ is determined by the number of data points, it is usually selected at about $\frac{1}{4}$ of the total data length. Typically, $F(n)$ will increase with box size $n$ [Fig. 2(c)]. A linear relationship on a double log graph indicates the presence of power-law (fractal) scaling. Under such conditions, the fluctuations can be characterized by a scaling exponent $\alpha$, the slope of the line relating $\log F(n)$ to $\log n$ [Fig. 2(c)].

As discussed above, a good linear fit (with $r>0.98$ ) of $\log F(n)$ vs. $\log n$ indicates that $F(n) \sim n^{\alpha}$, where $\alpha$ is the single exponent describing the correlation properties of the entire range of time scales. Figure 2(c) shows the DFA results for data from one young and one elderly subject. The scaling exponent ( $\alpha$ value) is reduced from 0.69 for the young adult to 0.58 for the elderly subject, indicating a shift from more complex fractal-like scaling to behavior that is more random. Note that $\alpha=0.5$ indicates that there is no correlation in the time series, i.e., white noise.

As described above, the DFA algorithm involves a detrending step. Our previous analysis ${ }^{10}$ showed that when the signal is highly nonstationary, it is necessary to apply higher-order detrending. In other words, instead of fitting the integrated signal with a linear trend, we can fit it with higher-order polynomials. ${ }^{10}$ In this study, we apply polynomials up to the fourth order. The results dis- cussed below are for parabolic detrending (polynomial of order 2). Higher-order polynomial detrending produced similar results.

\section{Power Spectrum Analysis}

A standard technique of time series analysis is spectral analysis. ${ }^{30,33}$ For time series with long-range correlations, the Fourier power spectrum shows power-law scaling behavior, i.e., the power spectrum is a straight line if plotted on a double-logarithmic graph. In other words, $S(f) \sim 1 / f^{\beta}$, where $S(f)$ is the Fourier power spectral density, $f$ denotes the frequency, and $\beta$ is the spectral scaling exponent.

In principle, the DFA scaling exponent $\alpha$ is related to the slope $\beta$ of the power spectrum $\log -\log$ plot by the equation: $\alpha=(1+\beta) / 2 .{ }^{29}$ Therefore, both DFA and Fourier analysis should lead to consistent results. However, in practice, for real-world signals that are nonstationary and noisy, Fourier analysis is less reliable than the DFA method for detecting long-range correlations. ${ }^{29}$

We derived power spectral density estimates by using two different Fourier analysis algorithms: (1) A standard fast Fourier transform algorithm for evenly sampled data; and (2) the Lomb Fourier transform for nonevenly sampled data. ${ }^{31}$ For the former case, we used the IBI time series indexed by the breathing cycle number. For the latter method, we used the actual time as the index for the IBI series, since these time series are unevenly sampled. Note that the units of the frequency are different in these two cases: $1 /$ cycle in the first method and 1/s $(\mathrm{Hz})$ for the Lomb method.

From the spectrum of each subject, a linear regression fit of power versus frequency on a $\log -\log$ scale was calculated [Fig. 2(d)]. To be consistent, the frequency range for the fit corresponds to the range of fit used in the DFA analysis.

\section{Assessing Effects of External Stimuli}

All subjects watched the movie Fantasia ${ }^{\odot}$ to help maintain wakefulness and provide a relatively constant environment during the $120 \mathrm{~min}$ data recording period. However, these common stimuli may influence breathing patterns of our subjects in a similar way. ${ }^{7}$

To determine whether the visual and auditory stimuli had a similar effect on different subject's breathing patterns, we measured the IBI cross correlation from pairs of subjects. The cross-correlation function, denoted as $\rho_{x y}(\tau)$, of two time series $x(t)$ and $y(t)$ is defined as

$$
\rho_{x y}(\tau)=\frac{E\left\{\left[x(t-\tau)-\mu_{x}\right]\left[y(t)-\mu_{y}\right]\right\}}{\sigma_{x} \sigma_{y}},
$$


TABLE 1. Interbreath interval dynamics. IBI: interbreath interval. DFA: detrended fluctuation analysis. FFT: fast Fourier transform. The IBI values are unadjusted means \pm SD.

\begin{tabular}{|c|c|c|c|c|}
\hline Variable & Young women & Young men & Elderly women & Elderly men \\
\hline Mean IBI (s) & $3.49 \pm 0.27$ & $3.96 \pm 0.76$ & $3.61 \pm 0.70$ & $3.83 \pm 0.57$ \\
\hline Range of IBI (s) & $3.03-3.89$ & $2.93-5.26$ & $2.60-4.87$ & $3.13-4.85$ \\
\hline$\alpha$ exponent by DFA & $0.70 \pm 0.07$ & $0.68 \pm 0.07$ & $0.67 \pm 0.06$ & $0.60 \pm 0.08^{\mathrm{a}}$ \\
\hline$\beta$ exponent by $\mathrm{FFT}^{\mathrm{b}}$ & $0.40 \pm 0.19$ & $0.35 \pm 0.14$ & $0.30 \pm 0.19$ & $0.23 \pm 0.20$ \\
\hline$\beta$ exponent by Lomb periodogram ${ }^{c}$ & $0.47 \pm 0.18$ & $0.39 \pm 0.19$ & $0.35 \pm 0.22$ & $0.25 \pm 0.27$ \\
\hline
\end{tabular}

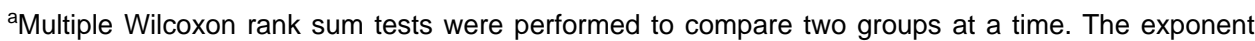
$\alpha$ for the group of elderly men is significantly different from the other three groups $(p<0.03$ for all three comparisons). The group average values of $\alpha$ are not statistically different among the groups of young men, young women, and elderly women ( $p>0.3$ for all comparisons).

${ }^{\mathrm{b}} \beta$ exponents derived by the FFT algorithm are not significantly different among groups.

${ }^{\mathrm{c}} \beta$ exponents derived by the Lomb algorithm are not significantly different among groups.

where $E[z]$ denotes the expectation value of variable $z$, and $\mu$ and $\sigma^{2}$ denote the mean and variance of the time series. In this analysis, $x(t)$ and $y(t)$ are averaged IBI time series (over $30 \mathrm{~s}$ ) to reduce noise.

Because the movie was not started at precisely the same moment after the beginning of each recording, the cross-correlation function was calculated with a variable lag time, $\tau$. The maximal value of this cross-correlation function is considered possibly meaningful if it is greater than two times the standard deviation of the crosscorrelation function calculated by using a randomized IBI time series (obtained by shuffling the original time series). We also recorded the corresponding time lag for which the maximal value of the cross-correlation function occurs.

We calculated all possible combinations of crosscorrelation functions. Of the total $780(=40 \times 39 / 2)$ pairs of subjects, only $3 \%$ had maximal values exceeding the threshold selected above. To select those values that likely represented meaningful cross correlations rather than chance effects, we applied a second criterion. If there is a common rhythm appearing in a certain subjects' breathing patterns, then a simple relationship among the time lags should exist. Let $\tau_{\mathrm{AB}}$ be the time lag for the maximal cross-correlation function for subjects $\mathrm{A}$ and $\mathrm{B}, \tau_{\mathrm{BC}}$ is the lag for subjects $\mathrm{B}$ and $\mathrm{C}$, and $\tau_{\mathrm{AC}}$ the lag for subjects $\mathrm{A}$ and $\mathrm{C}$. Then, we expect $\tau_{\mathrm{AB}}$ $+\tau_{\mathrm{BC}}=\tau_{\mathrm{AC}}$.

Only three subjects (1 young female, 1 elderly female, and 1 elderly male) showed evidence of common breathing patterns according to this second criterion. However, we observed that these three subjects had very different $\alpha$ exponents $(0.73,0.62$, and 0.56$)$, indicating that any movie-related breathing patterns probably had little effect on the long-range correlations.

In summary, cross-correlation analysis suggests that the movie had some effect on a small subset of subjects' respiration patterns. However, it did not seem to have a systematic effect on the correlation properties of the res- piration dynamics as measured by the DFA algorithm. This is probably due to the fact that the DFA algorithm removes slowly varying trends in the data.

\section{Statistical Analysis}

Subject characteristics and scaling exponents were compared between groups using the nonparametric Kruskal-Wallis test for statistically significant age/ gender effects (i.e., by stratifying all subjects by age and gender and testing for differences between the four groups of subjects). If this test showed a significant group effect on $\alpha$, multiple Wilcoxon rank sum tests were performed to compare two groups at a time (e.g., older men versus older women, young men versus older men) to test for age or gender effects. These nonparametric tests make no assumptions about the underlying distribution of the data being compared. Statistical analysis was performed using SAS software release 7.0 (Cary, North Carolina). A $p$ value less than 0.05 was considered statistically significant. Group results are reported as mean \pm standard deviation.

\section{RESULTS}

Tables 1 and 2 summarize the results for the mean and the range of IBI, as well as the scaling exponents of the IBI time series. The mean and range of IBI fluctuations did not show a significant dependence on age or gender.

Based on the DFA analysis, the fluctuations in the IBI time series were not simply attributable to random (uncorrelated) variations. Instead, we observed that scaling exponents $(\alpha \neq 0.5)$ are consistent with long-range correlations extending over a range of 10-400 breathing cycles. To verify that the long-range correlations were statistically significant, we did the following test. For each IBI time series, we randomized the sequential order of the IBI data, generating a surrogate time series with the same average and standard deviation as the original 
TABLE 2. Individual DFA exponents.

\begin{tabular}{|c|c|c|c|c|c|c|c|}
\hline \multicolumn{2}{|c|}{ Young female group } & \multicolumn{2}{|c|}{ Young male group } & \multicolumn{2}{|c|}{ Elderly female group } & \multicolumn{2}{|c|}{ Elderly male group } \\
\hline Age & $\alpha$ & Age & $\alpha$ & Age & $\alpha$ & Age & $\alpha$ \\
\hline 23 & 0.660 & 34 & 0.651 & 77 & 0.589 & 73 & 0.555 \\
\hline 28 & 0.646 & 31 & 0.680 & 73 & 0.622 & 81 & 0.527 \\
\hline 21 & 0.694 & 23 & 0.680 & 73 & 0.710 & 76 & 0.514 \\
\hline 30 & 0.735 & 21 & 0.602 & 71 & 0.618 & 71 & 0.572 \\
\hline 32 & 0.693 & 30 & 0.589 & 74 & 0.672 & 68 & 0.689 \\
\hline 23 & 0.649 & 23 & 0.654 & 73 & 0.644 & 83 & 0.565 \\
\hline 28 & 0.587 & 26 & 0.693 & 75 & 0.698 & 70 & 0.601 \\
\hline 27 & 0.748 & 31 & 0.667 & 85 & 0.734 & 77 & 0.624 \\
\hline 25 & 0.730 & 21 & 0.820 & 70 & 0.603 & 71 & 0.565 \\
\hline 21 & 0.845 & 21 & 0.783 & 73 & 0.777 & 77 & 0.768 \\
\hline mean $\pm S D$ & $0.70 \pm 0.07$ & & $0.68 \pm 0.07$ & & $0.67 \pm 0.06$ & & $0.60 \pm 0.08$ \\
\hline
\end{tabular}

data set. By definition, these surrogate time series are uncorrelated. As expected, DFA analysis showed that the $\alpha$ exponents were very close to 0.5 (with standard deviation less than 0.03 ) for these randomized time series. For each group of 10 subjects, the $\alpha$ values of the original IBI time series were then compared to the randomized time series. The Student's t test confirmed that the $\alpha$ exponents of all four groups were statistically different from those uncorrelated surrogate time series.

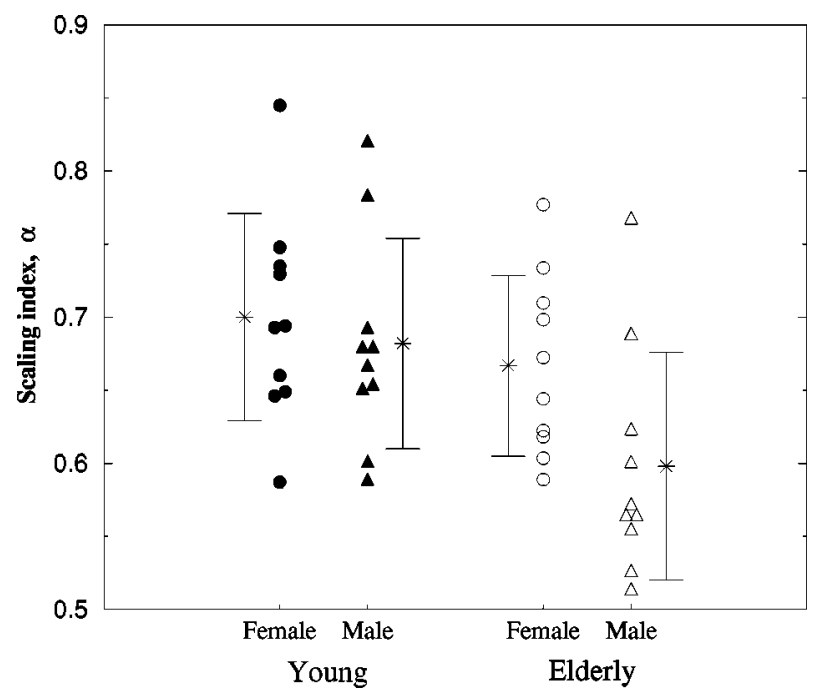

FIGURE 3. Scaling exponents $\alpha$ from DFA analysis for 40 subjects divided into four groups based on age and gender. The $\alpha$ values are obtained from the least-squares fit of the DFA analysis [see Fig. 2(c)] of the IBI time series over the range of 10 cycles (about $40 \mathrm{~s}$ ) to 400 cycles (about $26 \mathrm{~min}$ ). The asterisk with vertical bars denotes mean \pm S.D. for each group, respectively. The $\alpha$ values of the young female, young male, and elderly female groups are not statistically different from each other. The $\alpha$ exponents of the elderly male group are significantly different from the young male $(p<0.02)$, young female $(p<0.01)$, and elderly female $(p$ $=0.03)$ groups.
Next, each subject's $\alpha$ exponent was compared to $\alpha$ values of 100 surrogate time series (generated by 100 randomized representations of the original time series) to estimate the likelihood that the $\alpha$ exponent was different from that of its randomized surrogates. ${ }^{8}$ The DFA scaling exponents for 6 of the 10 elderly male subjects were not significantly different from that of the randomly shuffled (white-noise) control series. In contrast, the DFA scaling exponents from all subjects $(n=30)$ in the other three groups were significantly $(p<0.05)$ higher than the randomized control series.

The strength of the IBI fractal correlations appears to have both an age and gender dependence ( $p=0.02$ by the Kruskal-Wallis test). Figure 3 shows the results of DFA analysis of the IBI time series for all 40 subjects. The strength of correlation, as indicated by the $\alpha$ exponent, is significantly lower in the elderly male group but not in the elderly female group, compared to the young adults (Tables 1 and 2). Multiple Wilcoxon rank sum tests were performed to compare two groups at a time. The exponent $\alpha$ had a mean value of $0.60 \pm 0.08$ for the group of elderly men, significantly different from the other three groups $(p<0.03$ for all three comparisons). Moreover, 6 of the 10 elderly male subjects had $\alpha$ scaling exponents lower than those of any individual in the other groups. In contrast, the mean values of $\alpha$ were not statistically different among the groups of young men, young women, and elderly women.

The $\beta$ exponent (based on the $\log -\log$ slopes of the power spectrum) also showed a trend toward decreased values for the elderly male group (Tables 1 and 2), consistent with the $\alpha$ exponent changes. However, in contrast to DFA, neither of the spectral analysis methods [fast Fourier transform (FFT) or the Lomb periodogram method] showed significant age or gender differences in the power spectrum exponents. 


\section{DISCUSSION}

The results of this study are notable because they: (1) provide a new and easily applied algorithm for the detection of breathing cycle time series from noisy respiratory signals; (2) present consistent evidence for the presence of long-range (fractal) correlations in human respiratory dynamics, extending over hundreds of breathing cycles; and (3) present evidence that these correlations may degrade in elderly men. To our knowledge this is the first study specifically addressing possible longrange correlation properties in breath-to-breath dynamics in healthy adults and their possible alterations with aging.

The complex, nonstationary characteristics of these respiratory signals create important technical challenges related to reliably quantifying their behavior in the clinical setting. Therefore, it may be difficult to detect subtle changes in the dynamics of real-world data by power spectrum methods. Of note, in contrast to DFA, neither of the spectral analysis methods showed significant age or gender differences in the fractal scaling exponents. This apparent discrepancy is likely to be related to two factors: (1) The $\beta$ exponent derived from the Fourier transfer has been shown in previous studies to be less accurate than the DFA measurement for quantifying long-range correlations ${ }^{10,37}$ [Fig. 2(d)]. (2) Fourier analysis requires stationarity, which is likely to be an invalid assumption for the IBI time series. In contrast, the DFA takes into account certain types of nonstationary properties in the data.

A previous study by Hughson et al. ${ }^{15}$ using spectral analysis techniques, did raise the possibility of "fractal and/or deterministic chaos" in breathing dynamics of healthy young men $(n=8)$, but concluded that the "evidence is not strong." The present analysis was facilitated by the development of a new signal processing algorithm that does not alter the underlying dynamics of the respiration signal, and by employing a detrended fluctuation (fractal) analysis method. ${ }^{29}$

Fractal properties have been observed in the fluctuations of the depth of breathing (tidal volume) in anesthetized adult rats. ${ }^{13}$ In lambs, respiratory dynamics also acquire fractal scaling properties during fetal development. ${ }^{36}$ This presumably reflects the maturation of central nervous system respiratory control centers, chemoreflexes, pulmonary stretch reflexes, and neurohumoral influences that affect respiratory behavior over the short and long term. Aging is associated with neuronal dropout, loss of central signal integration, stiffening of the pulmonary parenchyma, and reduced chemoreceptor sensitivity. ${ }^{22}$

The loss of long-range correlations (multiscale organization) in breathing dynamics in humans is consistent with these physiologic alterations. Our findings lend pre- liminary support to this hypothesis in elderly men, but not women. These gender differences are not likely attributable to underlying differences in cardiovascular health, as both groups were carefully screened with noninvasive tests to help exclude clinically relevant cardiovascular disease. Since women experience greater longevity than men, these results may reflect subtle intrinsic gender differences in the rate at which physiological systems undergo degradative change. ${ }^{32}$ Whether this rate of physiological aging is primarily related to genetic or environmental factors remains to be determined. Further studies, at rest and with exercise, are needed to confirm our findings, and to determine whether women also undergo the loss of long-range respiratory correlations at a later age.

The finding of long-range correlations extending over hundreds of breathing cycles in healthy subjects also poses a challenge to ongoing efforts to model respiration dynamics. Previous studies have shown that beat-to-beat fluctuations of the healthy sinus rhythm also display long-range (fractal) correlations. ${ }^{17,29}$ In humans, fractal and nonlinear heart rate complexity also appears to degrade with age. ${ }^{17,18,21}$ Although the underlying mechanisms for the observed scaling behavior of breathing and heart rate dynamics, and other biologic signals, ${ }^{1,8,10,17}$ remain to be fully understood, the interaction over multiple time scales of different control and feedback systems probably plays an important role. ${ }^{9,23}$ However, these fractal correlation properties, and their changes with aging, are not explained by current models of neuroautonomic control.

Finally, the results of this study may also have practical implications for the design of ventilators. A recently described conceptual model has predicted that the addition of random noise to mechanical ventilation might improve gas exchange and prevent further lung injury, ${ }^{35}$ a finding supported by some experimental data. ${ }^{20,27}$ Whether a fractal pattern of respiratory variability, incorporating long-range correlations, will be of clinical value in further improving mechanical ventilation remains to be tested in experimental and clinical settings.

\section{ACKNOWLEDGMENTS}

This study was supported by grants from NIMH (MH54031), NIA (AG04390, AG14420, and an OAIC grant AG08812), NCRR (RR13622), the G. Harold and Leila Y. Mathers Charitable Foundation, the Fetzer Institute, and the Centers for Disease Control and Prevention. One of the authors (L.A.L.) holds the Irving and Edyth S. Usen and Family Chair in Geriatric Medicine at the Hebrew Rehabilitation Center for Aged. 


\section{REFERENCES}

${ }^{1}$ Bassingthwaighte, J. B., L. S. Liebovitch, and B. J. West. Fractal Physiology. New York: Oxford University Press, 1994.

${ }^{2}$ Beran, J. Statistics for Long-Memory Processes. New York: Chapman and Hall, 1994

${ }^{3}$ Buldyrev, S. V., A. L. Goldberger, S. Havlin, C.-K. Peng, M. Simons, and H. E. Stanley. Generalized Lévy walk model for DNA nucleotide sequences. Phys. Rev. E 47:4514-4523, 1993.

${ }^{4}$ Dejours, P., R. Puccinelli, J. Armand, and M. Dicharry. Breath-to-breath variations of pulmonary gas exchange in resting man. Respir. Physiol. 1:265-280, 1966.

${ }^{5}$ Edwards, R. D., and J. F. Magee. Technical Analysis of Stock Trends, 7th ed. Springfield: Amacom, 1997.

${ }^{6}$ Frey, U., M. Silverman, A. L. Barabasi, and B. Suki. Irregularity and power-law distributions in the breathing pattern in preterm and term infants. J. Appl. Physiol. 86:789-797, 1998.

${ }^{7}$ Haas, F., S. Distenfeld, and K. Axen. Effects of perceived musical rhythm on respiratory pattern. J. Appl. Physiol. 61:1185-1191, 1986.

${ }^{8}$ Hausdorff, J. M., C.-K. Peng, Z. Ladin, J. Y. Wei, and A. L. Goldberger. Is walking a random walk? Evidence for longrange correlations in the stride interval of human gait. $J$. Appl. Physiol. 78:349-358, 1995.

${ }^{9}$ Hausdorff, J. M., and C.-K. Peng. Multiscaled randomness: A possible source of $1 / f$ noise in biology. Phys. Rev. $E$ 54:2154-2157, 1996.

${ }^{10}$ Hausdorff, J. M., P. L. Purdon, C.-K. Peng, Z. Ladin, J. Y. Wei, and A. L. Goldberger. Fractal dynamics of human gait: Stability of long-range correlations in stride interval fluctuations. J. Appl. Physiol. 80:1448-1457, 1996.

${ }^{11}$ Hausdorff, J. M., S. L. Mitchell, R. Firtion, C.-K. Peng, M. E. Cudkowicz, J. Y. Wei, and A. L. Goldberger. Altered fractal dynamics of gait: Reduced stride interval correlations with aging and Huntington's disease. J. Appl. Physiol. 82:262-269, 1997

${ }^{12}$ Ho, K. K. L., G. B. Moody, C.-K. Peng, J. E. Mietus, M. G. Larson, D. Levy, and A. L. Goldberger. Predicting survival in heart failure cases and controls using fully automated methods for deriving nonlinear and conventional indices of heart rate dynamics. Circulation 96:842-848, 1997.

${ }^{13}$ Hoop, B., H. Krazemi, and L. Liebovitch. Rescaled range analysis of resting respiration. Chaos 3:27-29, 1993.

${ }^{14}$ Hughson, R. L., Y. Yamamoto, and J. O. Fortrat. Is the pattern of breathing at rest chaotic? A test of the Lyapunov exponent. Adv. Exp. Med. Biol. 393:15-19, 1995.

${ }^{15}$ Hughson, R. L., Y. Yamamoto, J.-O. Fortrat, R. Leask, and M. S. Fofana. Possible fractal and/or chaotic breathing patterns in resting humans. In: Bioengineering Approaches to Pulmonary Physiology and Medicine, edited by M. C. K. Khoo. New York: Plenum, 1996, pp. 187-196.

${ }^{16}$ Ivanov, P. Ch., M. Rosenblum, C.-K. Peng, J. Mietus, S. Havlin, H. E. Stanley, and A. L. Goldberger. Scaling behavior of heartbeat intervals obtained by wavelet-based time series analysis. Nature (London) 383:323-327, 1996.

${ }^{17}$ Iyenger, N., C.-K. Peng, R. Morin, A. L. Goldberger, and L. A. Lipsitz. Age-related alterations in the fractal scaling of cardiac interbeat interval dynamics. Am. J. Physiol. 271:R1078-R1084, 1996.

${ }^{18}$ Kaplan, D. T., M. I. Furman, S. M. Pincus, S. M. Ryan, L. A. Lipsitz, and A. L. Goldberger. Aging and the complexity of cardiovascular dynamics. Biophys. J. 59:945-949, 1991.
${ }^{19}$ Kolmogorov, A. N. Local structure of turbulence in fluid for very large Reynolds numbers. In Translations in Turbulence, edited by S. Friedlander and L. Topper. New York: Interscience, 1961, pp. 151-155.

${ }^{20}$ Lefevre, G. R., S. E. Kowalski, L. G. Girling, D. B. Thiessen, and W. A. C. Mutch. Improved arterial oxygenation after oleic acid lung injury in the pig using a computer-controlled mechanical ventilator. Am. J. Respir. Crit. Care Med. 154:1567-1572, 1996.

${ }^{21}$ Lipsitz, L. A., G. B. Mietus, G. B. Moody, and A. L. Goldberger. Spectral characteristics of heart rate variability before and during postural tilt. Relations to aging and risk of syncope. Circulation 81:1803-1810, 1990.

${ }^{22}$ Lipsitz, L. A. Clinical physiology of aging. In: Textbook of Internal Medicine, 3rd ed., edited by W. N. Kelley. Philadelphia: Lippincott, 1996, pp. 110-119.

${ }^{23}$ Lipsitz, L. A., S. M. Pincus, R. J. Morin, S. Tong, L. P. Eberle, M. Phyllis, and P. M. Gootman. Preliminary evidence for the evolution in complexity of heart rate dynamics during autonomic maturation in neonatal swine. J. Auton Nerv. Syst. 65:1-9, 1997.

${ }^{24}$ Mäkikallio, T. H., T. Seppänen, K. E. J. Airaksinen, J. Koistinen, M. P. Tulppo, C.-K. Peng, A. L. Goldberger, and H. V. Huikuri. Dynamic analysis of heart rate may predict subsequent ventricular tachycardia after myocardial infarction. Am. J. Cardiol. 80:779-783, 1997.

${ }^{25}$ Mandelbrot, B. B. The Fractal Geometry of Nature. San Francisco: Freeman, 1982.

${ }^{26}$ Montroll, E. W., and M. F. Shlesinger. The wonderful world of random walks. In: Nonequilibrium Phenomena II. From Stochastics to Hydrodynamics, edited by J. L. Lebowitz and E. W. Montroll. Amsterdam: North-Holland, 1984, pp. $1-121$.

${ }^{27}$ Mutch, W. A. C., S. Harms, M. R. Graham, S. E. Kowalski, L. G. Girling, and G. R. Lefevre. Biologically variable or naturally noisy mechanical ventilation recruits atelectatic lung. Am. J. Respir. Crit. Care Med. 162:319-323, 2000.

${ }^{28}$ Peng, C.-K., S. V. Buldyrev, S. Havlin, M. Simons, H. E. Stanley, and A. L. Goldberger. Mosaic organization of DNA nucleotides. Phys. Rev. E 49:1685-1689, 1994.

${ }^{29}$ Peng, C.-K., S. Havlin, H. E. Stanley, and A. L. Goldberger. Quantification of scaling exponents and crossover phenomena in nonstationary heartbeat time series. Chaos 5:82-87, 1995.

${ }^{30}$ Pomeranz, B., R. Macaulay, M. A. Caudill, I. Kutz, D. Adam, D. Gordon, K. M. Kilborn, A. C. Barger, D. C. Shannon, R. J. Cohen, and H. Benson. Assessment of autonomic function in humans by heart rate spectral analysis. Am. J. Physiol. 248:H151-H153, 1985.

${ }^{31}$ Press, W. H., B. P. Flannery, S. A. Teukolsky, and W. T. Vetterling. Numerical Recipes in C: The Art of Scientific Computing, 2nd ed. Cambridge, U.K.: Cambridge University Press, 1993

${ }^{32}$ Ryan, S. M., A. L. Goldberger, S. M. Pincus, J. E. Mietus, and L. A. Lipsitz. Gender and age-related differences in heart rate dynamics: Are women more complex than men? J. Am. Coll. Cardiol. 24:1700-1707, 1994.

${ }^{33}$ Simpson, D. M., and R. Wicks. Spectral analysis of heart rate indicates reduced baroreceptor-related heart rate variability in elderly persons. J. Gerontol. 43:M21-M24, 1988.

${ }^{34}$ Small, M., K. Judd, M. Lowe, and S. Stick. Is breathing in infants chaotic? Dimension estimates for respiratory patterns during quiet sleep. J. Appl. Physiol. 86:359-376, 1999.

${ }^{35}$ Suki, B., A. M. Alencar, M. K. Sujeer, K. R. Lutchen, J. J. Collins, J. S. Andrade, E. P. Ingenito, S. Zapperi, and H. E. 
Stanley. Life support system benefits from noise. Nature (London) 393:127-128, 1998.

${ }^{36}$ Szeto, H. H., P. Y. Cheng, J. A. Decena, Y. Cheng, D. L. Wu, and G. Dwyer. Fractal properties in fetal breathing dynamics.
Am. J. Physiol. 263:R141-R147, 1992.

${ }^{37}$ Taqqu, M. S., V. Teverovksy, and W. Willinger. Estimators for long-range dependence: An empirical study. Fractals 3:785-798, 1996. 